\title{
Líder o gerente, ¿qué los hace diferentes? (Leader or manager, What makes them different?)
}

\section{'Claudia Leticia Preciado-Ortiz iJosé Sánchez-Gutiérrez iiiJuan Antonio Vargas-Barraza}

\begin{abstract}
Over time, the concept of leadership has been analyzed from various perspectives and applied to many areas. Some analyze it from the human perspective, others from efficiency and productivity, some more define leadership styles and profiles. The present article is a theoretical analysis of the origin and evolution of leadership, types of leadership, as well as the differentiation between this term and that of management, that allows to establish the bases of later studies not only theoretical but also empirical with respect to the subject.
\end{abstract}

Key words: leader, leadership, management, manager.

JEL: M54, M50.

Resumen. A través del tiempo, el concepto de liderazgo ha sido analizado desde diversas perspectivas y aplicado a infinidad de áreas temáticas. Algunos lo analizan desde la visión humana, otros desde la eficiencia y productividad, algunos más definen estilos y perfiles de liderazgo. El presente artículo es un análisis teórico del origen, evolución y tipos de liderazgo, así como la diferenciación entre este término y el de gestión, que permita establecer las bases de posteriores estudios no sólo teóricos sino también empíricos respecto al tema.

Palabras clave: líder, liderazgo, gerente, gestión.

i Departamento de Mercadotecnia y Negocios Internacionales. Centro Universitario de Ciencias Económico Administrativas, Universidad de Guadalajara. Periférico Norte № 799, Núcleo Universitario Los Belenes, 45100 Zapopan, Jal., México. Correo electrónico: leticia.preciado@academicos.udg.mx

ii Departamento de Mercadotecnia y Negocios Internacionales. Centro Universitario de Ciencias Económico Administrativas, Universidad de Guadalajara, Periférico Norte № 799, Núcleo Universitario Los Belenes, 45100 Zapopan, Jal., México. Correo electrónico: jsanchez@cucea.udg.mx

iii Departamento de Mercadotecnia y Negocios Internacionales. Centro Universitario de Ciencias Económico Administrativas, Universidad de Guadalajara, Periférico Norte № 799, Núcleo Universitario Los Belenes, 45100 Zapopan, Jal., México. Correo electrónico: javb@cucea.udg.mx 


\section{Introducción}

El tema del liderazgo es tan amplio y tan diverso que difícilmente se podría recopilar y aún menos profundizar, en un pequeño trabajo, los diferentes enfoques y teorías a través de los que se ha abordado y analizado tanto su concepto, como tipos y aplicaciones a lo largo del tiempo. Históricamente hablando se tendría que comenzar por el análisis de la evolución del hombre, su vida en grupo y cómo desde la era primitiva, aún siendo nómada, vivía en familias o tribus y de manera instintiva organizaba estructuralmente el funcionamiento del grupo que les permitiera la supervivencia.

Desde ese momento ya se marcaba el perfil de un líder, cuyas características especiales determinaban la dirección de la tribu como tal, misma que, conforme fue evolucionando y deja de ser nómada, se apega a la necesidad de vivir en sociedad y organizarse, lo cual representó establecer estructuras jerárquicas más complejas que demandaban nombrar una cabeza que gobernara tanto al pueblo como así mismo.

De esta forma surge el liderazgo que se irá transformando a la par de la evolución humana y su vida en grupo; este liderazgo se verá reflejado en los grandes emperadores, reyes, guerreros, capataces, presidentes, etc. que han dejado grabado su nombre en la historia de la humanidad, y convirtiéndose en sujetos de estudio por su particular manera de gobernar y las contribuciones realizadas durante su poderío.

El presente documento tiene como objetivo realizar un análisis teórico del origen, evolución del liderazgo y tipos de liderazgo identificados, así como la diferenciación entre este término y el de gestión, con el fin de establecer las bases para posteriores estudios no sólo teóricos sino también empíricos respecto al tema aplicado a distintas líneas de investigación de la administración.

\section{Definición de liderazgo}

Existe una amplia bibliografía sobre la definición de las palabras liderazgo o líder, mismas que, en el transcurso de la historia, han tenido diferentes connotaciones y han sido analizadas desde diferentes perspectivas académicas; una de ellas es la historia. En ninguna otra disciplina se podrán encontrar ejemplos empíricos desde el comienzo de la civilización y los primeros registros existentes de cómo jefes de tribus, patriarcas, profetas, reyes, guerreros, mártires, gurús, capitanes, 
rebeldes y visionarios, entre otros, han marcado la existencia humana y, por ende, las actividades y transformación de un líder (Harter, 2015).

Según The Oxford English Dictionary el término líder se utilizó por primera vez en el siglo XIV y con el tiempo, el término liderazgo ha adquirido varios significados, cada uno de ellos enfocado en los distintos elementos del fenómeno como los seguidores, el proceso, el objetivo, el contexto, el propósito perseguido, etc. (Solomon, Costea \& Nita, 2016).

La palabra liderazgo tiene su historia de significado y cambio desde su origen (alrededor de 800 A.C.) a su evolución en el concepto de líder (alrededor de 1300 A.C.), y finalmente al liderazgo a mediados del siglo XIX (Grace, 2003).

Analizando la raíz etimológica de la palabra líder / liderazgo se encuentra literatura que la han analizado desde la perspectiva latina y la sajona.

Desde la etimología sajona, la palabra líder se verá como derivada del inglés leader, la cual es la que toma la Real Academia Española. Si se profundiza en la cuestión etimológica de dicha palabra, "leader" significa, en su carácter verbal y en términos generales, guiar por un camino, servir como indicador de ruta y ser un canal o conductor para algo (Grace, 2003).

Su raíz etimológica proviene de la palabra loedan, la forma casual de lithan, que significa viajar. Sin embargo, el Oxford English Dictionary señala que la definición de líder, muy relacionado con lo que hoy es liderazgo, apareció alrededor de 1225 con el significado "guiar con referencia a la acción y opinión, llevar por persuasión, conduje con argumentos, etc." (Grace, 2003).

De esta manera, líder significa, según la etimología de la lengua inglesa, el acto de guiar para avanzar e ir para adelante; definición que los hispanohablantes adoptaron alrededor de 1956 a través del proceso llamado barbarismo convirtiéndose en un anglicismo para el idioma español.

Desde la etimología latina, según el filósofo Hugo Landofi en su obra La esencia del liderazgo, expone que la palabra líder podría deber su origen en la partícula lid (nominativo latino), que proviene del latín lis-litis (genitivo latinos). Su definición original en la lengua latina es riña, disputa, proceso. Lo que deriva que líder no es un agente pasivo, sino como alguien con una postura activa en su realidad. Entonces, resumiendo, se puede decir que la palabra líder significa, etimológicamente hablando: persona activa en su comportamiento que guía a un grupo hacia un objetivo común.

Otras definiciones de liderazgo que se pueden encontrar son la de Goleman (2002, pág. 222), él menciona que "el liderazgo implica el arte de trabajar 
con la gente. Saber conducir no significa saber dominar, sino saber convencer a las personas de que trabajan por un objetivo común".

El profesor Mielu Zlate (2004) define el liderazgo como "el proceso mediante el cual una persona establece una meta o una dirección para una o un cierto número de personas, y determina que actúen con competencia y compromiso total con su realización".

El Oxford English Dictionary (2016) define la palabra liderazgo como "condición de líder". Y líder lo define como "persona que encabeza y dirige un grupo o movimiento social, político, religioso, etc.".

Por lo que se puede concluir que el líder es la persona que dirige a otras personas hacia un objetivo o meta común establecida trabajando en conjunto.

\section{Evolución del liderazgo}

La evolución del liderazgo se enmarca desde que el hombre primitivo se comienza a organizar y separar tareas para poder sobrevivir, viéndolo como si tuviera un desarrollo a la par de la administración. Al principio, cuando eran nómadas, se tenía un jefe de manada o tribu, los grupos debían ser pequeños porque eso facilitaba su traslado, aseguraba su alimentación, protección y supervivencia.

Conforme el hombre descubre la agricultura y se vuelve sedentario, comienza a surgir el estado y una organización más compleja donde la figura de líder se hizo más notoria, no porque no existía antes, sino porque la población empezó a crecer y hubo la necesidad de organizarse, definir un orden y una jerarquía. Aquí es donde nacen y se desarrollan las grandes civilizaciones como la sumeria, egipcia, babilónica, china, griega, romana y hebrea; que cada una con sus características específicas y el entorno en el que se desenvolvieron definieron estilos de liderazgo y trabajo que fueron marcando las pautas para las organizaciones actuales.

De aquí nos pasamos a la influencia de las organizaciones militares y la iglesia católica, llegando a la Edad Media con la etapa artesanal, los feudos, los mercaderes de Venecia, la ética protestante y todas las formas de organización que surgieron (además de ideologías como la de Maquiavelo y Sun Tzu) para culminar con la Revolución Industrial.

Una vez desarrollada la máquina de vapor, se comienza con la administración científica y el avance de otras disciplinas administrativas. Donde cada uno de los investigadores iba abonando desde su perspectiva a la cuestión de 
cómo administrar y, por defecto, liderar una empresa. Taylor, como padre de la administración científica; los esposos Gilberth por sus estudios de tiempos y movimientos; Henry Gantt, que fue el iniciador de las gráficas que llevan su nombre para calendarizar la producción. Pasando a la administración burocrática, que enfatiza en la necesidad de operar las empresas en forma racional y no en deseos arbitrarios de sus propietarios y gerentes.

Un caso es Max Weber, quien trató de visualizar cómo las grandes organizaciones, surgidas por la revolución industrial, podrían funcionar idealmente. Luego la escuela del proceso administrativo, con Henry Fayol, quien se centró en las actividades gerenciales y formuló los 14 principios para que permitieran hacer más eficiente las actividades internas de las organizaciones.

En 1938, Chester Barnard, en su libro Las funciones del ejecutivo, la teoría propone la aceptación de la autoridad, siendo la más conocida de sus contribuciones, donde establece que la autoridad efectiva depende en gran parte de una comunicación eficaz entre superiores y subordinados y ganarse el respeto de los empleados siempre y cuando se tomen en cuenta sus sentimientos y capacidades (Wolf, 1974). Comenzando así con los estudios sobre los factores que influían en el comportamiento humano, donde los primeros conductistas fueron: Hugo Münsterberg, Mary Parker Follet y Elton Mayo. Posteriormente se puede mencionar a Abraham Maslow, Herzberg y Douglas McGregor, cuyos estudios hablan de motivaciones y superación personal.

En 1961, W.C.H. Prentice rechaza la noción de liderazgo como el ejercicio de poder y fuerza o como una habilidad extraordinaria. Prentice definió liderazgo como "el logro de una meta mediante la dirección de colaboradores humanos" (pág. 2), y al líder exitoso, como aquel que puede comprender las motivaciones de la gente y lograr que todos los empleados participen haciendo confluir las necesidades e intereses individuales con el propósito del grupo. Establece que el poder, la fuerza, las amenazas de su conducta, su popularidad, etc. no son las características que hace que un gran líder tenga un logro excepcional; sino es la parte social y humana de su comportamiento, ya que sabe comprender a sus colaboradores y la relación entre las metas individuales de cada uno de ellos y la meta grupal que él debe llevar a cabo (Prentice, 1961).

En la actualidad, infinidad de teóricos han establecido el liderazgo desde diferentes aristas que han tratado de adaptar a un mundo cambiante y cada vez más exigente para el éxito de las organizaciones. 


\section{Tipos de liderazgo}

Así como las variables históricas, sociales y culturales han influido en la evolución del concepto del liderazgo, también han permitido identificar los tipos de líder que se han desarrollado conforme al entorno y la etapa cronológica de la historia (Giraldo y Naranjo, 2014), asociándolos a diversas teorías que se han desarrollado al respecto con diferentes enfoques y exponentes clave. Comenzando con la Teoría del Gran hombre, la Teoría de Rasgos (la rejilla gerencial), Teorías del comportamiento (líder autocrático o democrático), la Teoría de la contingencia (con los trabajos desarrollados por Friedler y Blanchard), la Teoría de la influencia (líder carismático) y finalmente la Teoría de las relaciones (Liderazgo transaccional y el transformacional) (Daft, 2006) (Véase figura 1).

La Teoría del Gran Hombre es la pionera en sentar las bases sobre estudios formales sobre liderazgo, centrándose en estudiar a los grandes líderes de la historia del mundo y concluyendo con la idea de que las personas nacían con ciertas características que las diferenciaba y les permitía ser líderes (los líderes nacen, no se hacen). Esta afirmación realizada por Thomas Carlyle, en 1840, lo hizo famoso. Sin embargo, 20 años después, en 1860, Herbert Spencer debatió tal teoría estableciendo que los grandes hombres de la historia eran producto del entorno que les tocó vivir y de las condiciones que les precedieron (Carlyle, 1840; Rives, 1912; Hook, 1950 y Carneiro, 1981).

Posteriormente, Ralph Stogdill, establece respecto a la Teoría de Rasgos con base en sus investigaciones, que no hay un grupo de características o cualidades coincidentes en las personas que les permitía identificarse como líderes, sino que éstas características se determinaban por situaciones específicas (Asociación Española de Coaching Ejecutivo - Organizativo y Mentoring, AECOP, 2006; Giraldo y Naranjo, 2014). Esto dio pie a que en diversas universidades se comenzaran a analizar las variables de éxito o fracaso de un líder, como fueron la Ohio State University, Harvard University y la Universidad de Michigan. Es entonces cuando empieza una tendencia a analizar el comportamiento del líder y su efectividad.

En 1964, Robert R. Blake y Jane Mouton desarrollan "el primer modelo de liderazgo denominado Malla gerencial" (Daft, 2006; citado en Giraldo y Naranjo, 2014, pág. 22). Es un modelo bidimensional donde el eje de las $X$ representa la atención a la producción y tareas y el eje de las $Y$ es la atención a las relaciones personales (ambos numerados del 1 al 9 , donde uno es muy baja atención y 9 
muy alta); evidenciando 81 estilos de liderazgo, donde los más predominantes son cinco estilos.

El primer estilo predominante lo nombraron como líder pobre o insuficiente, que es aquel poco interesado en la producción (tareas) y las relaciones, ubicado en el cuadrante $(1,1)$.

El segundo fue identificado como el liderazgo club campestre. Caracterizado por una alta tendencia hacia las relaciones pero muy poca atención a la producción (Cuadrante 1,9). En tercer lugar está el liderazgo equilibrado (Cuadrante $5,5)$, cuya característica es un enfoque moderado hacia ambos ejes, media entre la productividad y las relaciones.

El cuarto estilo es el que centra toda su atención en el cumplimiento de los objetivos pero descuida totalmente en la relación que tiene con el personal (Cuadrante 9,1). Finalmente, el quinto estilo se caracteriza por una fuerte atención tanto en la productividad como en las relaciones (Cuadrante 9,9).

Es aquí donde el análisis de estudio del liderazgo direcciona su atención hacia las acciones que lleva a cabo un líder, cómo actúa respecto a sus seguidores. Definiendo la Teoría del Comportamiento con base, según Daft (2006), en dos pilares: primeramente afirmando que un buen líder puede ser cualquiera que siga el comportamiento adecuado para tal responsabilidad, y segundo, que el comportamiento se puede aprender pero no los rasgos, por lo que el liderazgo está al alcance de todos. En esta etapa se identifican dos estilos de liderazgo: el democrático y autocrático, basados en la Teoría X y Y del comportamiento humano. En esta teoría se pueden identificar los investigadores Kart Lewin, Lippin y White, Robert Mc Murry, Rensis Likert, entre otros).

Continuando con la definición de los tipos de liderazgo, se encuentra la Teoría de la contingencia o situacional, que junta las características del líder, las relaciones con sus seguidores y la relación con el medio ambiente en el que se encuentran. Aquí resaltan el modelo de Fiedler o teoría de la efectividad del liderazgo, esta evalúa que el éxito depende de qué tan favorable es la situación en la que se encuentra el líder. Y el modelo de Hersey y Blanchard sobre liderazgo situacional propone que el liderazgo varía según las características de los colaboradores (Daft, 2006). Sin dejar de lado en esta parte de la historia las aportaciones de Tannenbaun y Schmidth; Evans y House; Kerr y Jermier; Vroom y Yetton; Fiedler \& García; Wofford.

Ahora damos lugar a las teorías emergentes. La primera es la teoría de la influencia cuyo enfoque básico es el del líder carismático, esta cualidad de 
carácter define su desempeño en la organización y su papel de liderazgo. Y en segundo lugar, la Teoría de las relaciones, la cual identifica al líder transaccional y al líder transformacional.

El liderazgo transaccional (de intercambio) se enfoca en identificar las necesidades de sus colaboradores y a través de recompensas se genera un vínculo para satisfacer las necesidades a cambio de cumplir con los objetivos organizacionales (Daft, 2006).

El liderazgo transformacional, un liderazgo ideal donde se alinean los objetivos y metas personales con las organizacionales. Se busca un cambio en los colaboradores a través de la inspiración, motivación y estimulación intelectual (Bass, 1981).

Tabla 1. Evolución de las teorías del liderazgo

\begin{tabular}{|c|c|c|}
\hline Teoría & Teoría & Enfoque \\
\hline \multicolumn{2}{|l|}{ Teoría del Gran Hombre } & Los líderes nacen no se hacen \\
\hline Teoría de los rasgos & Rejilla gerencial & Cualidades y atributos del líder \\
\hline \multirow[t]{2}{*}{ Teoría del comportamiento } & Liderazgo autocrático & $\begin{array}{l}\text { Rasgos y comportamientos del } \\
\text { líder }\end{array}$ \\
\hline & Liderazgo democrático & Seguidores \\
\hline \multirow[t]{2}{*}{ Teoría de la Contingencia } & $\begin{array}{l}\text { Modelo de Fiedler } \\
\text { Modelo situacional de Her- } \\
\text { sey y Blanchard }\end{array}$ & $\begin{array}{l}\text { Líder (Estilo, rasgos, comporta- } \\
\text { miento, posición) } \\
\text { Seguidores (Necesidades, ma- } \\
\text { durez, capacitación, cohesión) } \\
\text { Situación (Tarea, estructura, } \\
\text { sistemas, entorno) }\end{array}$ \\
\hline & & $\begin{array}{l}\text { Cualidades del líder carismático: } \\
\text { Amor por la vida, valoran a las }\end{array}$ \\
\hline Teoría de la Influencia & Liderazgo carismático & $\begin{array}{l}\text { personas, hacen el bien, dan } \\
\text { esperanza, estilo propio, com- } \\
\text { parten y piensan en los demás. }\end{array}$ \\
\hline Teoría de las relaciones & $\begin{array}{l}\text { Liderazgo transaccional } \\
\text { Liderazgo transformacional }\end{array}$ & Visión de futuro ideal \\
\hline
\end{tabular}

Fuente: Elaboración propia con base en Daft (2006)

La Tabla 1 muestra que la teoría más reciente va englobando los aspectos más benéficos y mejor desarrollados de sus antecesoras. De tal manera que si en un principio que creía que los líderes nacian, actualmente el líder se desarrolla conforme a su personalidad, contacto con sus seguidores y el entorno, de tal 
manera que le permita sobrevivir y sacar adelante la institución que dirija por muy grande o pequeña que sea.

\section{Liderazgo versus gestión}

Los términos "liderazgo" y "gestión" se usan a menudo con referencia al mismo concepto cuando,en realidad, son diferentes a pesar de que tienen muchos elementos comunes y actividades dirigidas a mejorar la organización. Por lo general, las diferencias se basan en el comportamiento personal del gerente y el líder, pero también en cómo logran transmitir sus ideas a las personas con las que trabajan.

Etimológicamente hablando, la palabra gestión tiene dos fuentes distintas. La primera es la palabra italiana meneggiare, que significa manejar las cosas, hacerse cargo de algo. $\mathrm{Y}$ a principios del siglo $\mathrm{XVI}$, se confundió con la palabra francesa menager, que significa uso cuidadoso (Toor, S., \& Ofori, G., 2008). Bavington (2005) observa que el término gestión abarca tres significados principales: la gestión como control (con raíces en la palabra latina manus), la gestión como cuidado (con raíces en la palabra francesa ménager), y la gestión como afrontamiento (una comprensión moderna de la gestión).

La definición actual de gestión en The Oxford English Dictionary es: "organización, supervisión o dirección; la aplicación de la habilidad de manipulación en el cuidado, uso, tratamiento o control de una cosa o persona, o en la conducta de algo" (Toor, S., \& Ofori, G., 2008). De igual forma The Oxford English Dictionary establece que gerente es la "persona que por oficio se encarga de dirigir, gestionar o adminsitrar una sociedad, empresa u otra entidad" (2016).

En 1977, la visión tradicional de la gestión y liderazgo se centraba en la estructura y los procesos de la organización, buscando un equilibrio entre competencia y control. Abraham Zaleznik pensaba que bajo este esquema los elementos esenciales del liderazgo como la inspiración, la visión y la pasión se omitían como parte del éxito empresarial.

Fue en este año cuando escribió Gerentes y líderes ison diferentes?, donde sostiene que los líderes de las empresas tienen más en común con los artistas, científicos y otros creativos que con los gerentes tradicionales. Sin dejar de reconocer que las organizaciones necesitan tanto gerentes como líderes, porque cada uno tiene una función particular, pero sin obstruir el trabajo entre ellos. Gerentes y líderes son personas muy distintas. 
Difieren en cuanto a su motivación, su historia personal y en su modo de pensar y actuar (Zaleznik, 1977).

Según Kotter (1990), en su artículo "Lo que de verdad hacen los líderes", describe a un líder muy relacionado a lo mencionado por Zaleznik tiempo antes. Kotter (1990) menciona que el liderazgo y la gestión son distintos y a la vez complementarios. Cada uno tiene su propia función y actividades características, sin embargo, ambos son necesarios para tener éxito en un entorno de negocios cada vez más complejo y volátil.

El verdadero desafío es combinar un liderazgo fuerte con una gestión fuerte, y usar a cada uno para equilibrar al otro. Desde luego, es comprensible que no todos pueden ser buenos en liderazgo y en gestión a la vez. Algunas personas tienen la capacidad de convertirse en excelentes ejecutivos, pero no en líderes fuertes. Otras tienen un gran potencial de liderazgo, pero por una serie de razones se les hace muy difícil convertirse en ejecutivos fuertes. Las empresas inteligentes valoran a los dos tipos de personas y trabajan duro para integrarlas a su equipo.

Daft (2003) contradice un poco esta idea ya que establece que todos los líderes son buenos gerentes pero que todos los gerentes tal vez no tengan cualidades de liderazgo.

Kotter (1990) puntualiza que las prácticas y procedimientos de la gestión son en gran medida una respuesta a uno de los acontecimientos más significativos del siglo XX: la aparición de las grandes organizaciones. Sin una buena gestión, las empresas complejas tienden a volverse de tal manera caóticas que incluso ponen en riesgo su propia existencia. Enfrentar la complejidad y enfrentar el cambio configuran las actividades principales de la gestión y el liderazgo, trabajando en equipo.

La Tabla 2 presenta de manera específica las diferencias documentadas por diversos autores en el tema sobre los términos de gestión y líderes, desde las primeras publicaciones de Zaleznik en 1977 hasta 2009 con Warren Bennis. 
Tabla 2. Diferencias entre líderes y gerentes

\section{Gerentes

- Se enfoca en la racionalidad y el control

- Se orienta hacia metas, recursos o personas

- $\quad$ Resuelve problemas

- Necesita perseverancia, determinación, trabajo duro, inteligencia, capacidad analítica, tolerancia y buena voluntad

- Tienen una actitud pasiva e impersonal

- Tienden a concebir el trabajo como un proceso que posibilita la interacción entre personas e ideas con el de establecer estrategias y tomar decisiones.

- Prefieren trabajar con personas. Evitan la actividad solitaria porque les genera ansiedad.

- Concentra su atención en cómo se hacen las cosas
- Se enfocan en enfrentar la complejidad.

- La buena gestión aporta un grado de orden y consistencia a dimensiones clave, como la calidad y la rentabilidad de los productos.

- La gestión desarrolla la capacidad para cumplir con su plan mediante la organización y la dotación de personal: creando una estructura organizacional y un conjunto de cargos para cumplir los requerimientos del plan, dotando a esos puestos con individuos calificados, comunicando el plan a esas personas, delegando la responsabilidad para la ejecución del plan, e ideando sistemas para monitorear su implementación.

- La gestión asegura el logro del plan mediante el control y la resolución de problemas: monitoreando con cierto detalle los resultados en relación al plan, tanto formal como informalmente, a través de informes, reuniones y otras herramientas; identificando desviaciones; y luego planificando y organizando la resolución de los problemas.

- Racionales, persistentes, resuelven problemas, analizan, estructuran, deliberan, decididos, analíticos, autoritarios y estabilizadores.

- Obtienen su poder de su posición y autoridad.
Adoptan una actitud personal y activa hacia las metas

Ejerce influencia al modificar estados de ánimo, evocar imágenes, expectativas y al establecer deseos y objetivos específicos Determina la dirección que toma el negocio. Desarrollan nuevos enfoques a problemas y están abiertos a nuevas alternativas Trabajan desde posiciones de alto riesgo Debido a su temperamento suelen buscar el riesgo y peligro, sobre todo cuando la oportunidad y la recompensa parecen prometedoras.

Se preocupan de las ideas, se relacionan de maneras más intuitivas y empáticas.

Se concentra en qué significan los acontecimientos y las decisiones para los participantes.

- Son muchas veces descritos con adjetivos cargados de contenido emocional.

Inspiran fuertes sentimientos de identidad o diferencia, de amor y odio.

- Las relaciones humanas en estructuras dominadas por líderes suelen ser turbulentas, intensas y por momentos hasta desorganizadas.

Se ocupa de enfrentar el cambio.

Liderazgo es alinear a las personas. Esto es comunicar la nueva orientación a quienes pueden crear coaliciones, comprenden la visión y están comprometidos con su logro. El logro de la visión requiere motivar e inspirar

Teoría de la contingencia 0 situacional

(Liderazgo efectivo y

relacional)

Fuente: Zaleznik, 1977

Teoría de la incluencia (líder carismético)

y Teoria las relaciones (Líder transaccional y transformacional)

Fuente: Kotter, 1990 y 2006

Son visionarios, apasionados, creativos, flexibles, inspiradores, innovadores, valiente, imaginativo, experimental, y promotor e iniciador de cambios.

Obtienen su poder de sus rasgos personales y atributos.

Hacen uso de su poder de referencia para influir en los seguidores.
Teoría de la influencia (Líder carismático, relacional)

Fuente: Capowski, 1994 


\begin{tabular}{|c|c|c|c|}
\hline & Gerentes & Líderes & $\begin{array}{l}\text { Enfoque de } \\
\text { estudio / fuente }\end{array}$ \\
\hline • & $\begin{array}{l}\text { Son administradores. } \\
\text { Desarrollan planes de negocios, presu- } \\
\text { puestos y monitorean su progreso. } \\
\text { Planean, presupuestan, evalúan. } \\
\text { Son más mente. }\end{array}$ & $\begin{array}{l}\text { - } \quad \text { Son agentes de cambio. } \\
\text { Buscan el cambio en personas y organiza- } \\
\text { ciones. } \\
\text { - Seleccionan talento, motivan, enseñan y } \\
\text { construyen confianza. } \\
\text { - Son más alma o corazón que mente. }\end{array}$ & $\begin{array}{l}\text { Teoría de la influencia } \\
\text { (Líder carismático, } \\
\text { relacional) } \\
\text { Fuente: Maccoby, } 2000\end{array}$ \\
\hline . & Tienen buena habilidad analítica. & Tienen buena intuición y visión & $\begin{array}{l}\text { Teoría de la influen- } \\
\text { cia y las relaciones } \\
\text { Fuente: Daft, } 2003\end{array}$ \\
\hline - & $\begin{array}{l}\text { Se movilizan por la posición de poder y la } \\
\text { autoridad. } \\
\text { Establecen sistemas, crean reglas y ope- } \\
\text { ran procedimientos y ponen en marcha } \\
\text { programas de incentivos. }\end{array}$ & $\begin{array}{l}\text { - Se movilizan por su poder personal y el res- } \\
\text { paldo del grupo. } \\
\text { Establecen una dirección, la comunican a } \\
\text { todos los que escuchan y mantendrán a la } \\
\text { gente motivada en tiempos difíciles. }\end{array}$ & $\begin{array}{l}\text { (Líder carismático, } \\
\text { relacional, transaccional y } \\
\text { transformacional) } \\
\text { Fuente: Robbins, } 2002\end{array}$ \\
\hline - & $\begin{array}{l}\text { Son visionarios, colaboradores, negocia- } \\
\text { dores y vendedores. }\end{array}$ & $\begin{array}{l}\text { Son capitanes, analistas, conductores y con- } \\
\text { troladores. }\end{array}$ & $\begin{array}{l}\text { Teoría de las relaciones } \\
\text { (Liderazgo transaccional) } \\
\text { Fuente: Zimmerman, } 2002\end{array}$ \\
\hline • & $\begin{array}{l}\text { Hacen que la empresa funcione a tiempo. } \\
\text { Se guían por el impulso miope para ma- } \\
\text { nejar la rutina con el fin de producir de } \\
\text { manera eficiente. } \\
\text { Son tácticos. }\end{array}$ & $\begin{array}{l}\text { - Deciden qué y a quién lleva la empresa y } \\
\text { hacia dónde se dirige. } \\
\text { Tienen amplias perspectivas que les per- } \\
\text { miten mirar en el futuro para determinar } \\
\text { necesidades y posibles cambios para el } \\
\text { crecimiento y supervivencia. } \\
\text { Son estrategas. }\end{array}$ & $\begin{array}{l}\text { Teoria de las relaciones } \\
\text { (Liderazgo transaccional) } \\
\text { Fuente: Perloff, } 2004\end{array}$ \\
\hline - & $\begin{array}{l}\text { Administra. } \\
\text { Es imagen. } \\
\text { Se mantiene. } \\
\text { Se centra en la estructura. } \\
\text { Se basa en el control. } \\
\text { La visión es a corto plazo. } \\
\text { Responde a las preguntas ¿qué? y } \\
\text { ¿cómo? } \\
\text { Se centra en resultados inmediatos. } \\
\text { Imita. } \\
\text { Acepta el status quo. } \\
\text { Hace las cosas como deben hacerse. }\end{array}$ & $\begin{array}{ll}\text { - } & \text { Innova. } \\
\text { - } & \text { Es original. } \\
\text { - } & \text { Se desarrolla. } \\
\text { - } & \text { Confía. } \\
\text { - } & \text { Su visión es a largo plazo. } \\
\text { - } & \text { El líder responde a ¿qué? } \\
\text { - } & \text { Se centra en resultados a mediano y largo } \\
& \text { plazo. } \\
\text { - } \quad \text { Crea. } \\
\text { - } \quad \text { Es desafiante. } \\
\text { Hace las cosas que deben hacerse. }\end{array}$ & $\begin{array}{l}\text { Teoría de las relaciones } \\
\text { (Lider transformacional) } \\
\text { Fuente: Bennis, } 2009\end{array}$ \\
\hline
\end{tabular}

Fuente: Elaboración propia.

Se observa que, independientemente del tiempo en el que se desarrolló el trabajo de investigación y los diferentes enfoques que los autores tenían para la realización del análisis y a partir del cual documentaron sus estudios, las coincidencias de lo que es un gerente y sus funciones así como el líder y sus funciones han estado claras y definidas.

Aunque haya muchas teorías sobre lo que es ser líder, queda claro que este necesita poseer un conjunto de características que deben ser aprendidas y/o desarrolladas, destacando la importancia de habilidades personales, facilidad de relación con personas y equipos de trabajo, comunicación efectiva, saber escuchar y resolver problemas; todo esto sin perder de vista el objetivo empresarial. 
Sin embargo, en donde entra la confusión de no poder diferenciar estos términos es en el mundo empresarial, ya en la práctica, donde la mayoría de las empresas creen que la cabeza del grupo (empresa de cualquier tipo) debe tener todas las cualidades y características de ambos, que sería lo ideal y es a donde todas las organizaciones deberían dirigirse en cuanto a formación de directivos de mandos medios hacia arriba, bajo el enfoque de cómo un gerente puede convertirse en líder y un líder pueda desempeñar las funciones de un gerente sin entrar en conflictos internos como persona ni con la organización ni con todos los demás elementos involucrados (empleados, proveedores, accionistas, etc.).

Se respalda este comentario con lo que Priestland y Hanig (2005) y Weathersby (1999) mencionan, es decir, que para explotar todo el potencial humano y los demás recursos, las organizaciones necesitarán desarrollar habilidades de liderazgo en sus ejecutivos y habilidades gerenciales en sus líderes. Cada vez hay más necesidad de liderazgo en todos los niveles de la organización y para cumplir con esa necesidad, los gerentes tienen que ser mejores en liderazgo (Toor, \& Ofori, 2008).

\section{Conclusión}

Es importante mencionar que las organizaciones deben adoptar estrategias para desarrollar sistemáticamente a sus administradores para que también sean líderes eficaces. Estos administradores, en determinadas circunstancias, pueden desempeñar un papel de liderazgo. Para este propósito, el desarrollo del liderazgo debe formar parte de la estrategia organizacional porque es una fuente de ventaja competitiva.

Prentice (1961) señala que un líder es exitoso cuando ha comprendido que los humanos son complejos y distintos, y que no sólo responden al estilo tradicional de dirección; sino también responden a cuestiones internas como amor, ambición, superación, pertenencia, aburrimiento, autoconfianza y muchas más. $Y$ que además la fuerza e importancia de estos intereses en cada persona es diferente y su grado de satisfacción también lo es.

Aunado a esto, Stogdill (1997) menciona que el liderazgo no puede surgir a menos que los miembros de un grupo asuman diferentes responsabilidades y que estas responsabilidades permitan satisfacer a cada miembro y se realicen mediante la comprensión de un interés o propósito mayor. Y por último, Bennis (1989), sostiene que llegar a ser un líder es sinónimo de convertirse en uno 
mismo; sin embargo, convertirse en un gerente se está convirtiendo en lo que una empresa quiere que se convierta. Los líderes producen el potencial de cambio dramático, caos e incluso fracaso; pero los gerentes producen estándares, consistencia, previsibilidad y orden (Kotter, 1990). Dependerá de la empresa que quiera tener: líderes o gerentes o ambos bien capacitados.

\section{Líneas de investigación a futuro}

Este trabajo de investigación es netamente un documento teórico realizado a través de la revisión exhaustiva de la bibliografía existente. Sin embargo, es posible el desarrollo de trabajos empíricos posteriores que permitan comprobar la diferencia o similitud entre los términos liderazgo y gestión; verificar si en el campo empresarial realmente se puede distinguir uno de otro, cuál es la percepción del empresario y del empleado al respecto y qué relación se puede identificar con los diferentes estilos de liderazgo y la gestión en una empresa. Considerando que es importante desarrollar un liderazgo ajustado al contexto, a la situación, al entorno, al tipo de colaboradores, e incluso al tipo de tarea que se realiza este es un campo amplio para la investigación en administración y planeación estratégica en todos los ámbitos, giros empresariales y más aún con el liderazgo transformacional que se considera el más completo e integral que tendrá que saber mezclar equilibradamente las relaciones personales y su bienestar sin perder de vista los objetivos y metas organizacionales. 


\section{Referencias}

Asociación Española de Coaching Ejecutivo-Organizativo y Mentoring (AECOP). (20122013). Breve historia del liderazgo. Recuperado de: http://aecop.net/es/articulos/ breve-historia-del-liderazgo/34

Bass, B. M. (1981). Stogdill's handbook of leadership: A Survey of theory and research, New York: Free Press.

Bavington, D. 2005. "Of fish and people: Managerial ecology in newfoundland and labrador cod fisheries." Unpublished dissertation, Ch.1, Wilfrid Laurier University, Waterloo, Ontario, 4-11, en https://www.researchgate.net/profile/Dean_Bavington/ publication/254728084_Of_fish_and_people_Managerial_ecology_in_Newfound land_and_Labrador_cod_fisheries/links/54fca5f70cf270426d102b9a/Of-fish -and-people-Managerial-ecology-in-Newfoundland-and-Labrador-cod-fisheries.pdf Bennis, W. 1989. On becoming a leader, Perseus, Reading, Mass.

Bennis, W., and Bennis, W. G. (2009), On becoming a leader. New York: Basic Books.

Capowski, G. 1994 . "Anatomy of a leader: Where are the leaders of tomorrow?" Manage. Rev., 83(3), 10-14.

Carlyle, T. (1840). The hero as divinity in: heroes and hero-worship. Yale University Press, en http://www.othroerirkindred.com/resources/Thomas $\% 20$ Carlyle $\% 20-\% 200$ n $\% 20$ Heroes $\% 2 \mathrm{C} \% 2 \mathrm{OHero}-$ Worship $\% 2 \mathrm{C} \% 20$ and $\% 20$ the $\% 20$ Heroic $\% 20$ in $\% 20$ History.pdf Carneiro, R. L. (1981). Herbert Spencer as an Anthropologist. Journal of Libertarian Studies, 5, 171-172.

Daft, R. (Ed.). (2006). La experiencia del liderazgo. México: CENGAGE Learning.

Daft, R. L. 2003. Management, 6, Ed., Dryden, London.

Giraldo, G. D. y Naranjo, A. J. (2014). Liderazgo: Desarrollo del concepto, evolución y tendencias. Estudio monográfico. Universidad del Rosario. Bogotá, Colombia, en http://repository.urosario.edu.co/bitstream/handle/10336/8672/1026275656-2014.pdf

Goleman, D. (2002), Emotional Intelligence. Bucharest: Curtea Veche.

Grace, M. 2003. "Origins of leadership: The etymology of leadership" Proc., International Leadership Association Conference, november 6-8, Guadalajara, Jalisco, México, en http://www.ila-net.org/publications/proceedings/2003/mgrace.pdf

Harter, N. (2015). Introduction-History in the study of leadership. Journal of Leadership Studies, 9(2), 39-41.

Hook, S. (1950) The hero in history, New York: Humanities Press, 67.

Kotter, J. P. (1990). What leaders really do. Harvard Bus. Rev., 5(3), 3-11.

Kotter, J. P. 2006. Leadership versus management: What's the difference? J. Qual. Participation, 29(2), 13-17.

Landofi, H. (2008). La esencia del liderazgo. Editorial Dunkan, en http://www.hugolandolfi. com/liderazgo.html

Maccoby, M. 2000. Understanding the difference between management and leadership. Res. Technol. Manag., 43(1), 57-59. 
Oxford English Dictionary (2016). En https://en.oxforddictionaries.com/?utm_source=odpanel\&utm_campaign=en

Prentice, W. C.H. (1961). Comprendiendo el liderazgo. Harvard Business Review, en https://issuu.com/elprofecharly1/docs/hrbal_-_lo_que_hacen_los_grandes_I_

Priestland, A., and Hanig, A. 2005 . "Developing first-level leaders". Harvard Bus. Rev., 83(6), 113-120.

Rives, R. (1912). La Monte Socialism: Positive and Negative, Chicago: Charles H. Kerr Publishing Company, 18.

Robbins, S. 2002. The difference between managing and leading, en http://www.Entrepreneur. com/article/ 0,462,304743,00.htm

Solomon, I. G., Costea, C., \& Niță, A. M. (2016). Leadership versus Managementin public organizations. Economics, Management \& Financial Markets, 11(1), 143-151.

Stogdill, R. 1997. Leadership, membership, and organization. Leadership: Classical, contemporary, and critical approaches, K. Grint, ed., Oxford University Press, 112-125.

Toor, S., \& Ofori, G. (2008). Leadership versus Management: How They Are Different, and Why. Leadership \& Management In Engineering, 8(2), 61-71.

Weathersby, G. B. 1999. Leadership versus management. Management Review, 88(5).

Wolf, William B. (1974). The basic Barnard: an introduction to Chester i. Barnard and his theories of organization and management. 1 Ed. Estados Unidos.

Zaleznik, A. 1977. Managers and leaders: Are they different? Harvard Bus. Rev., 55(3), 67-78.

Zimmerman, E. L. 2001. What's under the hood? The mechanics of leadership versus management. Healthcare Exec., 62(8), 10-12.

Zlate, M. (2004), Leadership and Management. Bucharest: Collegium. 